\title{
Cross-sectional and longitudinal comparisons of metabolic profiles between vegetarian and non-vegetarian subjects: a matched cohort study
}

\author{
Yen-Feng Chiu ${ }^{1 *}$, Chih-Cheng Hsu ${ }^{1}$, Tina H. T. Chiu ${ }^{2,3}$, Chun-Yi Lee ${ }^{1}$, Ting-Ting Liu ${ }^{4}$, \\ Chwen Keng Tsao ${ }^{4}$, Su-Chun Chuang ${ }^{1}$ and Chao A. Hsiung ${ }^{1}$ \\ ${ }^{1}$ Institute of Population Health Sciences, National Health Research Institutes, Zhunan, Miaoli County 35053, Taiwan \\ ${ }^{2}$ Medical Mission, Tzu Chi Foundation, Hualien 97002, Taiwan \\ ${ }^{3}$ Graduate Institute of Epidemiology and Preventive Medicine, National Taiwan University, Taipei 10017, Taiwan \\ ${ }^{4}$ MJ Health Management Institution, Taipei 10018, Taiwan
}

(Submitted 14 October 2014 - Final revision received 5 May 2015 - Accepted 7 July 2015)

\section{Abstract}

Several previous cross-sectional studies have shown that vegetarians have a better metabolic profile than non-vegetarians, suggesting that a vegetarian dietary pattern may help prevent chronic degenerative diseases. However, longitudinal studies on the impact of vegetarian diets on metabolic traits are scarce. We studied how several sub-types of vegetarian diets affect metabolic traits, including waist circumference, BMI, systolic blood pressure (SBP), diastolic blood pressure, fasting blood glucose, total cholesterol (TC), HDL, LDL, TAG and TC:HDL ratio, through both cross-sectional and longitudinal study designs. The study used the MJ Health Screening database, with data collected from 1994 to 2008 in Taiwan, which included 4415 lacto-ovo-vegetarians, 1855 lacto-vegetarians and 1913 vegans; each vegetarian was matched with five non-vegetarians based on age, sex and study site. In the longitudinal follow-up, each additional year of vegan diet lowered the risk of obesity by $7 \%$ (95\% CI 0.88, 0.99), whereas each additional year of lacto-vegetarian diet lowered the risk of elevated SBP by $8 \%$ ( $95 \%$ CI 0.85 , 0.99) and elevated glucose by $7 \%$ (95\% CI 0.87, 0.99), and each additional year of ovo-lacto-vegetarian diet increased abnormal HDL by $7 \%$ (95\% CI 1.03 1.12), compared with non-vegetarians. In the cross-sectional comparisons, all sub-types of vegetarians had lower likelihoods of abnormalities compared with non-vegetarians on all metabolic traits $(P<0 \cdot 001$ for all comparisons), except for HDL and TAG. The better metabolic profile in vegetarians is partially attributable to lower BMI. With proper management of TAG and HDL, along with caution about the intake of refined carbohydrates and fructose, a plant-based diet may benefit all aspects of the metabolic profile.

\section{Key words: Vegetarian diets: Longitudinal studies: Metabolic traits}

Metabolic abnormalities are key mechanistic steps in the development of coronary heart diseases ${ }^{(1)}$. Previous crosssectional studies in Western populations have suggested beneficial associations between a vegetarian diet and metabolic health $^{(2-6)}$, especially in cases where such a dietary pattern has been followed-up for 5 years or longer ${ }^{(7)}$.

Although several small cross-sectional studies have compared BMI, blood pressure, LDL oxidisability, fasting plasma, TAG, total cholesterol (TC) and LDL-cholesterol levels between Taiwanese vegetarians and matched omnivores ${ }^{(8,9)}$, examinations of the longitudinal effect of different types of vegetarian diets (e.g. vegan, lacto-ovo and lacto) on metabolic health are scarce. In addition, Western and Taiwanese vegetarian diets differ substantially, with Western vegetarian diets including more nuts, beans, dairy products, eggs and fresh greens, whereas Taiwanese vegetarians tend to eat more rice, cooked vegetables and soya products ${ }^{(3,4,8)}$. It is unknown whether the beneficial effect of vegetarian diets observed in Western populations is reproducible in Taiwanese vegetarians.

We conducted a matched cohort study to examine the effects of different types of vegetarian and non-vegetarian diets on metabolic traits in a large Taiwanese cohort. In addition, we compared the subjects' reported food intake from FFQ to provide information for speculation on the potential impact of these diets on metabolic profiles.

\section{Methods}

This study used the MJ Health Screening database, for which data were collected from 1994 to 2008 in Taiwan. The MJ Group runs the MJ Health Screening Center chain of private clinics, with health check-up clinics located in Taipei, Taoyuan, Taichung and Kaohsiung. Each clinic provides its members with periodic health examinations, which include anthropometric

Abbreviations: DBP, diastolic blood pressure; FBG, fasting blood glucose; MS, metabolic syndrome; NHRI, National Health Research Institutes; SBP, systolic blood pressure; TC, total cholesterol; WC, waist circumference.

* Corresponding author: Dr Y.-F. Chiu, email yfchiu@nhri.org.tw 
measures and a check of blood pressure and biochemical markers such as blood glucose and lipids. In addition, at the time of screening, each examinee completes a self-administered questionnaire about his or her socio-demographic and lifestyle characteristics, along with a 23-item $\mathrm{FFQ}^{(10)}$. The MJ Health Screening database includes 918718 check-up records from 473997 subjects. After excluding subjects whose records contained inconsistencies in age, sex, ID number, examination dates or death dates, along with smokers and those younger than 20 years of age, 315033 subjects remained (online Supplementary Tables S1 and S2). The remaining group included 4415 lacto-ovo-vegetarians, 1855 lacto-vegetarians and 1913 vegans. In order to control for potential confounding effects, we matched each vegetarian with five non-vegetarians based on age, sex and study site, resulting in a total of 49098 participants for the final analyses. This study was approved by the institutional ethics committees of the MJ Health Screening Center and the National Health Research Institutes (NHRI).

\section{Classification of dietary groups}

The dietary intakes were assessed through a self-administered FFQ. The FFQ remained the same from 1994 to 2008. The reliability and validity of the MJ Health Screening Center's FFQ have been evaluated previously ${ }^{(11,12)}$. The Spearman's correlation coefficients for two repeats of the FFQ within 1 year ranged from 0.29 to 0.47 (all $P<0.0001$ ) (online Supplementary Table S3). In addition, a summarised dietary quality index - the Overall Dietary Index, Revised, which is based on analysis of data from the FFQ - successfully predicted mortality in a cohort study, indicating the reproducibility of the FFQ ${ }^{(13,14)}$. The FFQ included twenty-three food items with specified serving sizes. For each item, the participants were asked to select one of the following five frequency categories that best characterised their eating habits over the past year: 'almost never', ' $1-3$ servings/ week', '4-6 servings/week', ' 1 serving/d' or ' $\geq 2$ servings/d'(15). Participants were categorised into the following four diet categories - vegan, lacto-vegetarian, lacto-ovo-vegetarian and omnivore - based on the answers to the following questions: (1) Are you a vegetarian? (2) How much meat (including red meat, poultry and fish) do you eat? (3) How much dairy do you consume? (4) How many eggs do you eat? Participants who answered the first and the second questions inconsistently were excluded. Moreover, participants with multiple visits were excluded from the longitudinal analysis if their answers for these questions were inconsistent during different visits, but were included for cross-sectional comparison using data from the latest visit. A total of 6690 subjects were included in the longitudinal follow-up, with an average follow-up of $2 \cdot 12$ years. The follow-up times for each diet groups are presented in online Supplementary Table S4.

\section{Metabolic traits}

The metabolic traits evaluated in this study included waist circumference (WC), BMI, systolic blood pressure (SBP), diastolic blood pressure (DBP), fasting blood glucose (FBG), TAG, TC, HDL, LDL and the TC:HDL ratio. Blood was collected after overnight fasting on the entry date. FBG, TAG, HDL, TC and LDL were measured using an auto-analyser (Hitachi 7150; Hitachi). The presence of metabolic risk components was determined using the modified National Cholesterol Education Program/Adult Treatment Panel III (NCEP/ATP III) criteria: $\mathrm{WC} \geq 90 \mathrm{~cm}$ in men or $\geq 80 \mathrm{~cm}$ in women, TAG $\geq 1.69 \mathrm{mmol} / 1$ or on medication, HDL-cholesterol $<1.03 \mathrm{mmol} / \mathrm{l}$ in men or $<1.29 \mathrm{mmol} / 1$ in women, blood pressure $>130 / 85 \mathrm{mmHg}$ or on medication, $\mathrm{TC} \geq 5.17 \mathrm{mmol} / \mathrm{l}$ or on medication and FBG $\geq 5.56 \mathrm{mmol} / 1$ or on medication. The modified ATP III criteria have been shown to be predictive of mortality and CVD in Asians and Taiwanese in previous studies ${ }^{(16)}$. BMI $\geq 27$ was also included because it has been shown to be a risk factor for metabolic-related diseases for the Taiwanese population ${ }^{(17)}$.

\section{Statistical analysis}

Baseline characteristics were analysed using conditional logistic regression analyses. Generalised estimating equation (GEE) approaches were used to evaluate both the cross-sectional and temporal effects of different types of vegetarian diets relative to the omnivore diet for the dichotomised metabolic traits ${ }^{(18)}$. The 'logit' link was used for dichotomous outcomes, and an exchangeable correlation matrix was assumed in the GEE analyses. Covariates included age, sex, leisure-time physical activity (LTPA), alcohol consumption and education. Age $^{2}$ was also adjusted for in the traits when it was significant. Physical activity was presented as the metabolic equivalent of task (MET), where 1 MET equals $4.19 \mathrm{~kJ} / \mathrm{kg}$ per $\mathrm{h}(1 \mathrm{kcal} / \mathrm{kg}$ per $\mathrm{h}$ ) of body weight ${ }^{(19)}$. MET is defined as the ratio of the work metabolic rate to a standard RMR of $4.19 \mathrm{~kJ} / \mathrm{kg}$ per h ( $1.0 \mathrm{kcal} / \mathrm{kg}$ per h). One MET is considered as the RMR or the energy cost of a person at rest $^{(20)}$. The GEE approach accounting for within-subject corrections from repeat measurements is applicable to data under the missing completely at random missing mechanism ${ }^{(18)}$. The non-parametric locally weighted scatterplot smoothing (LOESS) method $^{(21)}$ was used to depict the patterns of individual quantitative traits over age; participants on medications (or with diseases) related to these traits were excluded. The relative quantity of food intakes of different vegetarian groups compared with non-vegetarians was assessed by linear regression using the GEE approach, while adjusting for the same covariates stated above. The response variable was the food intake at multiple times, and the covariates of interest were the diet groups indicated by three dummy variables. All the statistical analyses were carried out using SAS 9.3.

\section{Results}

Table 1 shows the baseline characteristics of vegetarians and matched non-vegetarians. Vegetarians had significantly lower abnormal rates for WC (21.7 v. 27.7\%), BMI (10.9 v. 15.4\%), $\operatorname{SBP}(35 \cdot 3 v \cdot 40 \cdot 2 \%), \operatorname{DBP}(27 \cdot 3 v \cdot 32 \cdot 0 \%), \operatorname{FBG}(32 \cdot 0 v .37 \cdot 6 \%)$, TC (29.9 v. 50.0\%) and LDL (23.4 v. 42.3\%) levels, along with lower TC:HDL ratios (15.9 v. 21.0\%) (all $P<0.0001$ ), but higher abnormal rates for TAG (24.8 v. 23.0\%, $P=0.0003)$ and HDL (41.0 v. 34.8\%, $P<0.0001$ ), compared with non-vegetarians. 
Table 1. Comparison of baseline characteristics in vegetarians and matched non-vegetarians (Mean values and standard deviations; percentages)

\begin{tabular}{|c|c|c|c|c|c|c|}
\hline \multirow[b]{2}{*}{ Variables } & \multicolumn{2}{|c|}{ Vegetarian ( $n$ 8183) } & \multicolumn{3}{|c|}{ Matched non-vegetarian ( $n 40915$ ) } & \multirow[b]{2}{*}{$P^{*}$} \\
\hline & Mean & SD & Mean & & SD & \\
\hline Male (\%) & \multicolumn{2}{|r|}{$25 \cdot 0$} & \multicolumn{3}{|c|}{$25 \cdot 0$} & 1 \\
\hline Age (years) & 48.9 & 14.4 & 48.9 & & 14.4 & 0.16 \\
\hline \multicolumn{7}{|l|}{ Age $(\%)$} \\
\hline $20-39$ & \multicolumn{2}{|r|}{29.8} & \multicolumn{3}{|c|}{29.8} & 1 \\
\hline $40-54$ & \multicolumn{2}{|r|}{$32 \cdot 6$} & \multicolumn{3}{|c|}{$32 \cdot 6$} & \\
\hline $55-64$ & \multicolumn{2}{|r|}{$22 \cdot 1$} & \multicolumn{3}{|c|}{$22 \cdot 1$} & \\
\hline $65-98$ & \multicolumn{2}{|r|}{$15 \cdot 5$} & \multicolumn{3}{|c|}{$15 \cdot 5$} & \\
\hline WC $(\mathrm{cm})$ & \multirow{2}{*}{\multicolumn{2}{|c|}{$21 \cdot 7$}} & 77.1 & & $10 \cdot 0$ & $<0.0001$ \\
\hline WC $($ male $\geq 90$, female $\geq 80, \%$ ) & & & \multicolumn{3}{|c|}{$27 \cdot 7$} & $<0.0001$ \\
\hline BMI $\left(\mathrm{kg} / \mathrm{m}^{2}\right)$ & \multirow[t]{2}{*}{$22 \cdot 8$} & 3.4 & \multirow[t]{2}{*}{$23 \cdot 5$} & & 3.6 & $<0.0001$ \\
\hline $\mathrm{BMI}(\geq 27, \%)$ & & $10 \cdot 9$ & & $15 \cdot 4$ & & $<0.0001$ \\
\hline $\mathrm{SBP}(\mathrm{mmHg})$ & \multirow[t]{2}{*}{$122 \cdot 5$} & $21 \cdot 9$ & \multirow[t]{2}{*}{$125 \cdot 1$} & & $22 \cdot 1$ & $<0.0001$ \\
\hline $\operatorname{SBP}(\geq 130, \%)$ & & $35 \cdot 3$ & & $40 \cdot 2$ & & $<0.0001$ \\
\hline $\mathrm{DBP}(\mathrm{mmHg})$ & \multirow[t]{2}{*}{$72 \cdot 0$} & $12 \cdot 0$ & 73.9 & & $12 \cdot 0$ & $<0.0001$ \\
\hline $\mathrm{DBP}(\geq 85, \%)$ & & $27 \cdot 3$ & & $32 \cdot 0$ & & $<0.0001$ \\
\hline FBG $(\mathrm{mmol} / \mathrm{l})$ & 5.54 & 1.38 & $5 \cdot 66$ & & 1.48 & $<0.0001$ \\
\hline FBG $(\geq 5 \cdot 56, \%)$ & & $32 \cdot 0$ & & 37.6 & & $<0.0001$ \\
\hline TAG $(\mathrm{mmol} / \mathrm{l})$ & 1.37 & 0.93 & 1.35 & & 1.00 & 0.048 \\
\hline TAG $(\geq 1.69, \%)$ & & $24 \cdot 8$ & & $23 \cdot 0$ & & 0.0003 \\
\hline $\mathrm{TC}(\mathrm{mmol} / \mathrm{l})$ & 4.73 & 0.98 & $5 \cdot 23$ & & 0.99 & $<0.0001$ \\
\hline TC $(\geq 5.17, \%)$ & & 29.9 & & $50 \cdot 0$ & & $<0.0001$ \\
\hline $\mathrm{HDL}(\mathrm{mmol} / \mathrm{l})$ & 1.33 & 0.37 & 1.40 & & 0.40 & $<0.0001$ \\
\hline $\mathrm{HDL}($ male $<1.03$, female $<1.29, \%)$ & & $41 \cdot 0$ & & 34.8 & & $<0.0001$ \\
\hline $\operatorname{LDL}(\mathrm{mmol} / \mathrm{l})$ & 2.79 & 0.82 & 3.24 & & 0.86 & $<0.0001$ \\
\hline $\operatorname{LDL}(\geq 3 \cdot 36, \%)$ & & $23 \cdot 4$ & & $42 \cdot 3$ & & $<0.0001$ \\
\hline TC:HDL ratio & 3.8 & $1 \cdot 2$ & 4.0 & & 1.3 & $<0.0001$ \\
\hline TC:HDL ratio $(\geq 5, \%)$ & & $15 \cdot 9$ & & 21.0 & & $<0.0001$ \\
\hline LTPA (MET-h, \%) & & & & & & \\
\hline Inactive & & $49 \cdot 0$ & & $49 \cdot 6$ & & 0.28 \\
\hline Low & & $19 \cdot 6$ & & $19 \cdot 7$ & & \\
\hline Median & & $19 \cdot 8$ & & 19.4 & & \\
\hline High & & 7.9 & & $7 \cdot 4$ & & \\
\hline Very high & & 3.7 & & 3.9 & & \\
\hline Alcohol consumption (\%) & & & & & & \\
\hline Non-drinker & & $92 \cdot 3$ & & $85 \cdot 1$ & & $<0.0001$ \\
\hline Occasional & & 7.4 & & $13 \cdot 6$ & & \\
\hline Regular & & 0.3 & & 1.3 & & \\
\hline Education (\%) & & & & & & \\
\hline Middle school or below & & $46 \cdot 3$ & & 44.6 & & 0.0003 \\
\hline High school or junior college & & 34.7 & & $35 \cdot 6$ & & \\
\hline College or higher & & $19 \cdot 0$ & & 19.8 & & \\
\hline Site & & & & & & \\
\hline Taipei & & 43.5 & & 43.5 & & 1 \\
\hline Taoyuan & & 24.4 & & 24.4 & & \\
\hline Taichung & & $14 \cdot 1$ & & $14 \cdot 1$ & & \\
\hline Kaohsiung & & 18.0 & & 18.0 & & \\
\hline
\end{tabular}

WC, waist circumference; SBP, systolic blood pressure; DBP, diastolic blood pressure; FBG, fasting blood glucose; TC, total cholesterol; LTPA, leisure-time physical activity (MET: $1 \mathrm{MET}=4.19 \mathrm{~kJ} / \mathrm{kg}$ per h $(1 \mathrm{kcal} / \mathrm{kg} \mathrm{per} \mathrm{h}$ ) of body weight; MET-h, product of intensity (MET) and duration of exercise (h).

* $P$-values for matched data were derived from conditional logistic regression.

For LTPA, there was no difference between vegetarians and non-vegetarians $(P=0.28)$; however, vegetarians were more likely not to drink alcohol $(92.3 v .85 \cdot 1 \%, P<0.0001)$ and had a lower educational level $(46.3 v .44 .6 \%$ for middle school or lower education, $P=0 \cdot 0003)$. All significant differences between the dietary patterns have been reported; however, a number of those differences, although significant, was only small in magnitude and could have been caused by the large study sample. Fig. 1 illustrates the metabolic patterns over age by dietary type. Non-vegetarians had higher levels of all metabolic traits than vegetarians, except for TAG. Most metabolic traits increased with age until the age of 60 years, except for HDL. At approximately the age of 60 years, the trend decreased or stabilised for DBP, TC, LDL and BMI. The differences in BMI, LDL and TC between the omnivores and the vegetarian groups were constantly higher across all ages, whereas SBP, DBP, FBG, TAG, HDL and WC as well as TC:HDL ratios showed interactions with dietary types.

With adjustment for age, sex, physical activities, alcohol consumption and education, vegetarians had significantly lower abnormalities in WC, BMI, SBP, DBP, FBG, TC and LDL as well as in TC:HDL ratios, with OR ranging from 0.37 to 0.90 , but higher abnormality in HDL (OR ranged from $1 \cdot 17$ to $1 \cdot 52$ ), when compared with non-vegetarians cross-sectionally (Table 2 ). The 

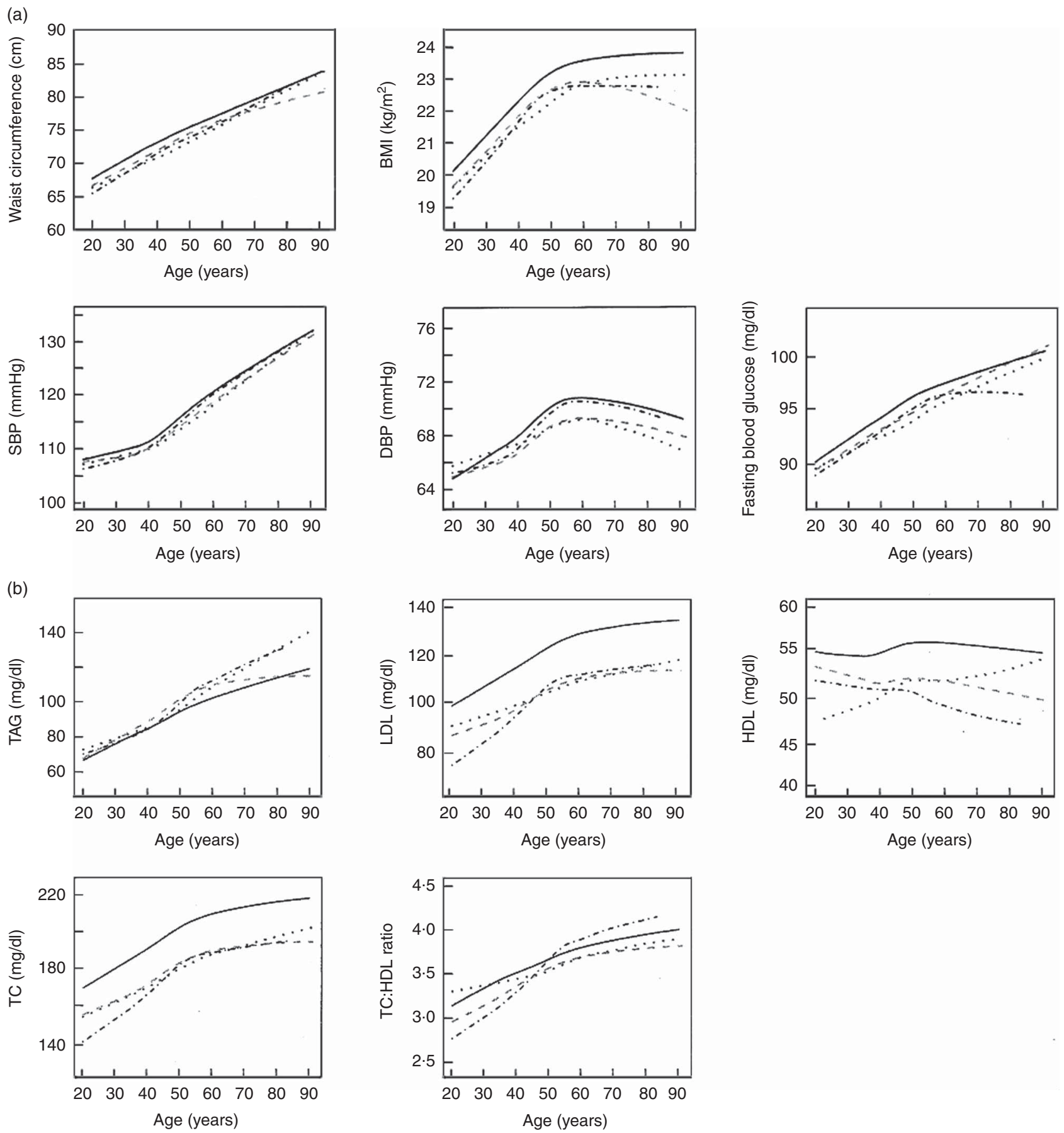

Fig. 1. (a) Patterns of waist circumference, BMI, systolic blood pressure (SBP), diastolic blood pressure (DBP), and fasting blood glucose over age by dietary type. (b) The patterns of TAG, LDL, HDL, total cholesterol (TC) and TC:HDL ratios over age by dietary type. - . - ., Vegan; . . . ., lacto; - - - ovo-lacto; _ - omnivore. To convert fasting blood glucose from $\mathrm{mg} / \mathrm{dl}$ to $\mathrm{mmol} / \mathrm{l}$, multiply by 0.0555 . To convert TAG from $\mathrm{mg} / \mathrm{dl}$ to $\mathrm{mmol} / \mathrm{l}$, multiply by 0.0113 . To convert $\mathrm{LDL}$, HDL and total cholesterol from $\mathrm{mg} / \mathrm{dl}$ to $\mathrm{mmol} / \mathrm{l}$, multiply by 0.0259 .

proportions of abnormal TAG appeared to be higher in lactoovo-vegetarians compared with non-vegetarians (OR 1.15; $95 \%$ CI 1.06, 1.24), whereas no significant difference was seen for vegans (OR 1.11; 95\% CI 0.99, 1.24) or lacto-vegetarians (OR $1.07 ; 95 \%$ CI $0.95,1 \cdot 19)$. Although vegetarians were more likely to have abnormal HDL levels, lacto and lacto-ovo-vegetarians had better TC:HDL ratios compared with non-vegetarians (lacto vegetarians: OR 0.79; $95 \%$ CI 0.69, 0.90; lacto-ovo-vegetarians: OR 0.58; $95 \%$ CI 0.52, 0.64). With additional adjustment for BMI (Table 3), the beneficial effects for blood pressure and blood glucose were partly attenuated, whereas the effect on lipids remained consistent.

The 1-year temporal effects of a vegetarian diet generally showed beneficial trends for metabolic traits (Table 2), even after 
Table 2. Longitudinal dietary effects on metabolic traits without adjusting for BMI (Odds ratios and $95 \%$ confidence intervals)

\begin{tabular}{|c|c|c|c|c|c|c|c|c|c|c|c|c|c|c|}
\hline \multirow[b]{3}{*}{ Abnormality of metabolic variable } & \multicolumn{8}{|c|}{ Baseline assessment } & \multicolumn{6}{|c|}{ Temporal effect } \\
\hline & \multicolumn{2}{|c|}{ Omnivore } & \multicolumn{2}{|c|}{ Vegan $v$. omnivore } & \multicolumn{2}{|c|}{ Lacto $v$. omnivore } & \multicolumn{2}{|c|}{ Ovo-lacto v. omnivore } & \multicolumn{2}{|c|}{ Vegan $v$. omnivore } & \multicolumn{2}{|c|}{ Lacto $v$. omnivore } & \multicolumn{2}{|c|}{ Ovo-lacto $v$. omnivore } \\
\hline & OR & $95 \% \mathrm{Cl}$ & OR & $95 \% \mathrm{Cl}$ & OR & $95 \% \mathrm{Cl}$ & OR & $95 \% \mathrm{Cl}$ & OR & $95 \% \mathrm{Cl}$ & OR & $95 \% \mathrm{Cl}$ & OR & $95 \% \mathrm{Cl}$ \\
\hline$W C(\geq 90 / 80 \mathrm{~cm}) \dagger$ & 1.01 & $1.00,1.02$ & 0.69 & $0.61,0.78$ & 0.62 & $0.55,0.70$ & 0.68 & $0.62,0.74$ & 0.98 & $0.89,1.09$ & 0.98 & $0.90,1.06$ & 0.98 & $0.93,1.03$ \\
\hline BMI $\left(\geq 27 \mathrm{~kg} / \mathrm{m}^{2}\right)^{*}$ & 1.03 & $1.02,1.03$ & 0.68 & $0.59,0.78$ & 0.60 & $0.52,0.71$ & 0.69 & $0.62,0.76$ & 0.93 & $0.88,0.99$ & 0.96 & $0.89,1.03$ & 0.96 & $0.91,1.00$ \\
\hline $\mathrm{SBP}(\geq 130 \mathrm{mmHg}) \dagger$ & 1.04 & $1.03,1.05$ & 0.81 & $0.73,0.90$ & 0.71 & $0.63,0.79$ & 0.76 & $0.70,0.82$ & 1.00 & $0.91,1.09$ & 0.92 & $0.85,0.99$ & 0.98 & $0.93,1.03$ \\
\hline $\mathrm{DBP}(\geq 85 \mathrm{mmHg})^{\star}$ & 1.03 & $1.03,1.04$ & 0.81 & $0.73,0.91$ & 0.69 & $0.62,0.78$ & 0.76 & $0.70,0.82$ & 1.03 & $0.94,1 \cdot 13$ & 0.97 & $0.89,1.06$ & 0.99 & $0.94,1.04$ \\
\hline FBG $(\geq 5.56 \mathrm{mmol} / /) \dagger$ & 1.09 & $1.08,1.09$ & 0.82 & $0.74,0.91$ & 0.73 & $0.65,0.81$ & 0.77 & $0.72,0.83$ & 1.01 & $0.93,1.10$ & 0.93 & $0.87,0.99$ & 0.98 & $0.94,1.02$ \\
\hline TAG $(\geq 1.69 \mathrm{mmol} / \mathrm{l}) \dagger$ & 1.02 & $1.01,1.02$ & 1.11 & $0.99,1.24$ & 1.07 & $0.95,1.19$ & $1 \cdot 15$ & $1 \cdot 06,1 \cdot 24$ & 0.98 & $0.90,1.08$ & 1.01 & $0.94,1.09$ & 1.03 & $0.99,1.08$ \\
\hline $\mathrm{TC}(\geq 5.17 \mathrm{mmol} / \mathrm{l}) \dagger$ & 1.02 & $1.01,1.02$ & 0.40 & $0.36,0.45$ & 0.40 & $0.36,0.45$ & 0.39 & $0.37,0.42$ & 0.96 & $0.89,1.05$ & 1.04 & $0.97,1.12$ & 0.99 & $0.94,1.03$ \\
\hline $\mathrm{HDL}(<1.03 / 1.29 \mathrm{mmol} / \mathrm{l}) \dagger$ & 0.92 & $0.92,0.93$ & 1.52 & $1.39,1.68$ & 1.42 & $1.29,1.57$ & $1 \cdot 17$ & $1 \cdot 10,1.25$ & 1.03 & $0.94,1.12$ & 0.98 & $0.90,1.08$ & 1.07 & $1.03,1.12$ \\
\hline LDL $(\geq 3.36 \mathrm{mmol} / \mathrm{l}) \dagger$ & 0.98 & $0.98,0.99$ & 0.45 & $0.40,0.50$ & 0.43 & $0.39,0.48$ & 0.37 & $0.34,0.40$ & 0.91 & $0.81,1.03$ & 1.01 & $0.93,1.09$ & 1.01 & $0.95,1.06$ \\
\hline TC:HDL ratio $(\geq 5) \dagger$ & 0.93 & $0.92,0.94$ & 0.90 & $0.80,1.02$ & 0.79 & $0.69,0.90$ & 0.58 & $0.52,0.64$ & 0.94 & $0.79,1 \cdot 12$ & 0.98 & $0.83,1.15$ & 1.07 & $1.00,1.15$ \\
\hline
\end{tabular}

WC, waist circumference; SBP, systolic blood pressure; DBP, diastolic blood pressure; FBG, fasting blood glucose; TC, total cholesterol; LTPA, leisure-time physical activity. * Adjusted for age, age ${ }^{2}$, sex, education, LTPA, alcohol consumption and study site.

† Adjusted for age, sex, education, LTPA, alcohol consumption and study site.

Table 3. Longitudinal dietary effects on metabolic traits with adjustment for BMI

(Odds ratios and $95 \%$ confidence intervals)

\begin{tabular}{|c|c|c|c|c|c|c|c|c|c|c|c|c|c|c|}
\hline \multirow[b]{3}{*}{ Abnormality of metabolic variable } & \multicolumn{8}{|c|}{ Baseline assessment } & \multicolumn{6}{|c|}{ Temporal effect } \\
\hline & \multicolumn{2}{|c|}{ Omnivore } & \multicolumn{2}{|c|}{ Vegan $v$. omnivore } & \multicolumn{2}{|c|}{ Lacto $v$. omnivore } & \multicolumn{2}{|c|}{ Ovo-lacto $v$. omnivore } & \multicolumn{2}{|c|}{ Vegan $v$. omnivore } & \multicolumn{2}{|c|}{ Lacto $v$. omnivore } & \multicolumn{2}{|c|}{ Ovo-lacto $v$. omnivore } \\
\hline & OR & $95 \% \mathrm{Cl}$ & OR & $95 \% \mathrm{Cl}$ & OR & $95 \% \mathrm{Cl}$ & OR & $95 \% \mathrm{Cl}$ & OR & $95 \% \mathrm{Cl}$ & OR & $95 \% \mathrm{Cl}$ & OR & $95 \% \mathrm{Cl}$ \\
\hline$W C(\geq 90 / 80 \mathrm{~cm}) \dagger$ & 0.99 & $0.98,1.00$ & 1.04 & $0.87,1.24$ & 0.84 & $0.71,1.01$ & 0.86 & $0.76,0.97$ & 1.06 & $0.90,1.25$ & 0.94 & $0.82,1.07$ & 0.99 & $0.92,1.07$ \\
\hline $\operatorname{SBP}(\geq 130 \mathrm{mmHg}) \dagger$ & 1.04 & $1.03,1.04$ & 0.90 & $0.80,1.00$ & 0.79 & $0.70,0.89$ & 0.84 & $0.77,0.91$ & 1.01 & $0.93,1.11$ & 0.92 & $0.85,1.01$ & 0.98 & $0.93,1.03$ \\
\hline DBP $(\geq 85 \mathrm{mmHg})^{\star}$ & 1.03 & $1.02,1.04$ & 0.90 & $0.81,1.02$ & 0.77 & $0.68,0.87$ & 0.83 & $0.76,0.90$ & 1.05 & $0.96,1.15$ & 0.98 & $0.90,1.08$ & 0.99 & $0.94,1.04$ \\
\hline $\mathrm{FBG}(\geq 5.56 \mathrm{mmol} / \mathrm{l}) \dagger$ & 1.08 & $1.08,1.09$ & 0.89 & $0.80,0.99$ & 0.79 & $0.71,0.88$ & 0.83 & $0.77,0.89$ & 1.02 & $0.93,1.12$ & 0.94 & $0.87,1.01$ & 0.98 & $0.94,1.03$ \\
\hline TAG $(\geq 1.69 \mathrm{mmol} / \mathrm{l}) \dagger$ & 1.01 & $1.00,1.02$ & 1.29 & $1 \cdot 15,1.45$ & 1.26 & $1 \cdot 12,1.42$ & 1.33 & $1 \cdot 22,1.44$ & 1.00 & $0.91,1.10$ & 1.02 & $0.95,1.11$ & 1.04 & $0.99,1.09$ \\
\hline $\mathrm{TC}(\geq 5.17 \mathrm{mmol} / \mathrm{l}) \dagger$ & 1.01 & $1.01,1.02$ & 0.42 & $0.38,0.46$ & 0.43 & $0.38,0.47$ & 0.41 & $0.38,0.44$ & 0.97 & $0.89,1.06$ & 1.05 & $0.98,1.13$ & 0.99 & $0.94,1.03$ \\
\hline $\mathrm{HDL}(<1.03 / 1.29 \mathrm{mmol} / \mathrm{l}) \dagger$ & 0.91 & $0.91,0.92$ & 1.72 & $1.56,1.89$ & 1.63 & $1.47,1.80$ & 1.30 & $1.21,1.39$ & 1.04 & $0.95,1.14$ & 0.99 & $0.90,1.10$ & 1.08 & $1.03,1.12$ \\
\hline $\operatorname{LDL}(\geq 3.36 \mathrm{mmol} / \mathrm{l}) \dagger$ & 0.98 & $0.97,0.99$ & 0.47 & $0.42,0.52$ & 0.46 & $0.41,0.51$ & 0.38 & $0.35,0.41$ & 0.92 & $0.82,1.04$ & 1.01 & $0.93,1.10$ & 1.01 & $0.95,1.06$ \\
\hline TC:HDL ratio $(\geq 5) \dagger$ & 0.92 & $0.91,0.93$ & 1.01 & $0.89,1.15$ & 0.90 & $0.79,1.03$ & 0.63 & $0.57,0.70$ & 0.96 & $0.80,1.16$ & 0.98 & $0.82,1.18$ & 1.08 & $1.00,1.16$ \\
\hline
\end{tabular}

WC, waist circumference; SBP, systolic blood pressure; DBP, diastolic blood pressure; FBG, fasting blood glucose; TC, total cholesterol; LTPA, leisure-time physical activity.

*Adjusted for age, age ${ }^{2}$, sex, BMI, education, LTPA, alcohol consumption and study site.

t Adjusted for age, sex, BMI, education, LTPA, alcohol consumption and study site. 
Table 4. Food intake frequency for different types of vegetarians compared with non-vegetarians (Estimates and $95 \%$ confidence intervals)

\begin{tabular}{|c|c|c|c|c|c|c|}
\hline \multirow[b]{2}{*}{ Food category } & \multicolumn{2}{|c|}{ Vegan } & \multicolumn{2}{|c|}{ Lacto } & \multicolumn{2}{|c|}{ Ovo-lacto } \\
\hline & Estimate $^{*}$ & $95 \% \mathrm{Cl}$ & Estimate* $^{\star}$ & $95 \% \mathrm{Cl}$ & Estimate* $^{\star}$ & $95 \% \mathrm{Cl}$ \\
\hline Rice and noodles & -0.27 & $-0.56,0.022$ & -0.21 & $-0.50,0.072$ & 1.00 & $0.82,1 \cdot 18$ \\
\hline Whole grains & 0.76 & $0.59,0.92$ & 0.85 & $0.69,1.00$ & 0.71 & $0.61,0.81$ \\
\hline Sweet breads & -0.68 & $-0.77,-0.59$ & -0.15 & $-0.25,-0.041$ & -0.098 & $-0.16,-0.034$ \\
\hline Beans & 1.04 & $0.87,1.20$ & 1.50 & $1.33,1.67$ & 1.66 & $1.56,1.76$ \\
\hline Light-green vegetables & 0.75 & $0.59,0.91$ & 0.89 & $0.74,1.05$ & 0.75 & $0.66,0.84$ \\
\hline Dark-green vegetables & 0.66 & $0.51,0.82$ & 0.81 & $0.66,0.96$ & 0.71 & $0.63,0.80$ \\
\hline Root vegetables & 0.20 & $0.10,0.31$ & 0.40 & $0.30,0.51$ & 0.50 & $0.44,0.56$ \\
\hline Fried rice and noodle products & -0.087 & $-0.16,-0.014$ & 0.016 & $-0.069,0.10$ & $0 \cdot 16$ & $0 \cdot 10,0.21$ \\
\hline Stir-fried rice or noodles & -0.28 & $-0.41,-0.15$ & 0.058 & $-0.10,0.22$ & -0.0075 & $-0.17,0.16$ \\
\hline Fried vegetables & -1.99 & $-2 \cdot 66,-1 \cdot 33$ & 0.14 & $-0.57,0.85$ & -0.30 & $-0.70,0.10$ \\
\hline Fried food & -0.80 & $-0.87,-0.73$ & -0.61 & $-0.69,-0.53$ & -0.49 & $-0.55,-0.44$ \\
\hline Fruit & -0.14 & $-0.26,-0.012$ & 0.087 & $-0.039,0.21$ & -0.21 & $-0.28,-0.13$ \\
\hline Jam and honey & $-0 \cdot 11$ & $-0.15,-0.069$ & 0.13 & $-0.069,0.19$ & 0.10 & $0.063,0.14$ \\
\hline Sugar drinks & -0.71 & $-0.84,-0.58$ & -0.51 & $-0.64,-0.38$ & -0.31 & $-0.40,-0.22$ \\
\hline Pickled vegetables & -0.36 & $-0.42,-0.30$ & -0.28 & $-0.34,-0.21$ & -0.23 & $-0.27,-0.19$ \\
\hline Instant noodles & -0.070 & $-0.10,-0.038$ & 0.014 & $-0.029,0.056$ & 0.040 & $0.012,0.067$ \\
\hline
\end{tabular}

* Servings/week, adjusted for age, sex, education, leisure-time physical activity, alcohol consumption and study site.

adjusting for the baseline metabolic profile. However, perhaps due to the limited sample size of individuals with multiple follow-up visits, only three differences in the temporal dietary effects were observed. First, each additional year of vegan diet lowered the risk of obesity by $7 \%$ (95\% CI 0.88, 0.99). Second, each additional year of lacto-vegetarian diet lowered the risk of elevated SBP by $8 \%(95 \%$ CI $0.85,0.99)$ and lowered the risk of elevated glucose by $7 \%$ (95\% CI 0.87, 0.99). Finally, each additional year of ovo-lacto-vegetarian diet increased abnormal HDL by $7 \%(95 \%$ CI $1.03,1.12)$ compared with a nonvegetarian diet. Nevertheless, overall TC:HDL ratios in ovolacto-vegetarians remained lower than that in non-vegetarians because they had the lowest baseline-level values.

With additional adjustment for BMI (Table 3), the abnormal rates of TAG at baseline became significantly higher in vegans and lacto-vegetarians compared with non-vegetarians, with OR of $1.29 ; 95 \%$ CI $1.15,1.45$ and OR of $1.26 ; 95 \%$ CI $1.12,1.42$, respectively. Although lacto-ovo-vegetarians appeared to have an increased 1-year temporal risk for a high TC:HDL ratio compared with non-vegetarians (OR 1.08; $95 \%$ CI 1.00, 1.16), this is largely due to their lower baseline TC:HDL ratio; the lacto-ovo-vegetarians actually had a lower overall average TC: HDL ratio compared with non-vegetarians.

Furthermore, we examined the intake of twenty plant-based foods (servings/week) for the four dietary groups at baseline (Table 4). Vegans had the lowest intake of fried foods, fried rice and noodle products, fried vegetables, jam and honey, instant noodles and pickled vegetables. Lacto-vegetarians had the highest intake of whole grains, light-green and dark-green vegetables and fruits. Ovo-lacto-vegetarians had the highest intake of beans and root vegetables. Non-vegetarians had the highest intake of (sweet) bread, fried vegetables, fried food, sugary drinks and pickled vegetables.

The summary of abnormality rates for individual metabolic traits at each visit are displayed in the supplemental materials (online Supplementary Tables S5 and S6). Visit frequencies, by diet and the number of participants and missing observations for each abnormal metabolic trait, are listed in online Supplementary Table S7 and S8. The numbers of subjects included in the analyses for Tables 2-4 are displayed in online Supplementary Tables S9-S11, respectively.

\section{Discussion}

Overall, we observed lower values for WC, BMI, SBP, DBP, FBG, TC, HDL and LDL, along with lower TC:HDL ratios, in vegetarians compared with non-vegetarians, which replicated the findings of previous Taiwanese studies ${ }^{(9,22,23)}$. Except for HDL and TAG values in lacto-ovo-vegetarians, the vegetarian diets showed significant beneficial effects on metabolic traits, which may be partly due to the lower BMI of vegetarians.

Similar to previous Taiwanese studies, our cross-sectional analysis indicates that vegetarians consistently showed a better lipid profile, except for TAG and HDL, although longitudinal follow-up showed no significant associations, suggesting potential reverse causation in which individuals with poor health conditions switched to a vegetarian diet in order to improve their health. Previous studies have found that higher TAG levels may be caused by high consumption of refined carbohydrates, fructose or fat ${ }^{(24)}$; however, generally, vegetarians in our study, consumed lower amounts of sugary drinks, sweet bread and fried food and, except for lacto-ovo-vegetarians, higher amounts of vegetables and whole grains (healthier type of carbohydrates). Lacto-ovo-vegetarians appeared to eat more carbohydrates and fructose, which could be one of the main causes for TAG elevation in this group. The dietary choices of vegetarians indicate that they may be more health conscious; such behaviours may have been motivated, in part, by the need to improve health in the presence of existing chronic degenerative conditions. Whether the lower HDL in vegetarians can be regarded as a risk factor may require further 
study, as vegetarians generally had better TC:HDL ratios. In addition, previous studies have found that low HDL due to reduced fat intake was not associated with poor cardiovascular health $^{(25,26)}$.

Several studies in Western countries have found that vegetarian diets play a favourable role in lowering the risk of metabolic syndrome (MS) ${ }^{(2,27-29)}$. However, similar to the results of Shang et al. ${ }^{(15)}$, in the present study, the prevalence of MS, defined by modified ATP III criteria for Asians ${ }^{(15)}$, did not differ between vegetarians and non-vegetarians, except for lacto-vegetarians (OR 0.87; 95\% CI 0.76, 0.99). When the International Diabetes Federation's criteria for MS (which requires elevated WC plus two additional risk factors) was used, all types of vegetarians showed a significantly lower MS prevalence compared with non-vegetarians (OR 0.76; $95 \% \mathrm{CI}$ $0.65,0.89$; OR 0.67; $95 \%$ CI 0.57, 0.78; OR 0.78; $95 \%$ CI 0.70 , 0.87 for vegans, lacto-vegetarians and lacto-ovo-vegetarians, respectively), with adjustment for age, alcohol consumption, LTPA and education. This indicates that the beneficial effects of a vegetarian diet may be mainly mediated through lower likelihood of obesity and abdominal obesity. Recently, the Adventist Health Study 2 also found that a vegetarian dietary pattern is associated with a more favourable MS profile ${ }^{(2)}$ and greater longevity ${ }^{(30)}$. The slight discrepancy in our findings may result partially from differences between Taiwanese and Western diets in food intakes and cooking styles, and differences between the two populations in the prevalence of obesity and abdominal obesity.

Our finding on blood pressure is consistent with both the EPIC-Oxford study and the Adventist Health Study 2, which found that vegetarians, particularly vegans, had a lower ageadjusted prevalence of hypertension and lower blood pressure compared with non-vegetarians; these differences were largely attributable to the lower BMI of the vegetarians ${ }^{(31)}$. In addition, we found that 1 additional year of exposure to lacto-vegetarian diet (instead of a non-vegetarian diet) is associated with reduced chance of elevated blood pressure. Lacto-vegetarians had the highest intake of whole grains, fruits and vegetables foods that are high in $\mathrm{K}$ - which are known to reduce blood pressure.

The EPIC-Oxford study showed that vegetarians had a significantly lower risk of hospitalisation or death due to IHD compared with non-vegetarians ${ }^{(31)}$. The Adventist Health Study 2 also found that vegetarians had lower cardiovascular mortality than non-vegetarians ${ }^{(32)}$. Whether this MJ Health Screening cohort replicates these findings deserves further investigation.

The limitations of this study are that the participants came back irregularly and were re-evaluated at unequal time intervals. Of the participants, $63.2 \%$ had only one visit recorded. Sensitivity analysis on those with only one visit and those with multiple visits showed no significant differences in the baseline associations of the separate subsets. An assumption of constant diet patterns was made when the dietary types were confirmed by the FFQ and when the answers to the specific dietary questions remained consistent over multiple visits. A longer duration ( $\geq 2$ decades) of very low meat intake has been shown to be associated with an increase in life expectancy of 3.6 years ${ }^{(30)}$. In this study, access to complete dietary duration information was not feasible, and longer dietary effects will be assessable with a longer follow-up period. As the MJ Health Screening Center requires an annual fee, its enrollees tend to be better off than the general population. Overall, they represent a group that has a higher socioeconomic status and is more health conscious than the general population. However, the participants of the MJ Health Screening Center are comparable with subjects attending other health-examination clinics ${ }^{(11,12)}$. Their diets may represent those of the middle-to-upper class in society ${ }^{(33)}$. However, some of the statistically significant findings may appear by chance due to multiple testing for multiple traits. Even with such limitations, this study remains one of the largest Taiwanese samples of vegetarians and omnivores to examine the longitudinal effects of vegetarian diets in Taiwan. These findings were mostly consistent with previous studies on Taiwanese vegetarians $^{(22,23)}$. Lacto-ovo-vegetarians, who are the majority of Taiwanese vegetarians, did not appear to have a more advantageous metabolic profile than the other two types of vegetarians. On the contrary, based on the FFQ, vegans appeared to have a healthier food profile than the others. These observations suggest that, with a cautious management of carbohydrate and fructose intake ${ }^{(34)}$, plant-based diets can benefit all aspects of the metabolic profile.

\section{Acknowledgements}

The authors are grateful to the reviewers for their constructive and insightful comments, which greatly improved the quality of this manuscript. The authors would like to thank Drs Wen-Harn Pan and Hsing-Yi Chang for their valuable suggestions. The authors also thank Ms Su-Mei Wang and Li-Feng Huang for their great efforts in the analysis. Finally, the authors thank all MJ participants of this study and Mr Mark Swofford (Scientific Editing Office, NHRI) for his dedicated contribution in editing this manuscript.

This work was supported by the grants PH-101-PP-04, PH-102-PP-04, PH-103-PP-04 and PH-104-PP-04 from the NHRI in Taiwan. The NHRI had no role in the design, analysis or writing of this article.

Y.-F. C., C.-C. H., T. H. T. C. and S.-C. C. designed and wrote the paper; C.-Y. L. analysed the data; and T.-T. L., C. K. T. and C. A. H. conducted the research.

There are no conflicts of interest to declare.

\section{Supplementary material}

For supplementary material/s referred to in this article, please visit http://dx.doi.org/doi:10.1017/S0007114515002937

\section{References}

1. Simons LA, Simons J, Friedlander Y, et al. (2011) Is prediction of cardiovascular disease and all-cause mortality genuinely driven by the metabolic syndrome, and independently from its component variables? The Dubbo study. Heart Lung Circ 20, 214-219. 
2. Rizzo NS, Sabate J, Jaceldo-Siegl K, et al. (2011) Vegetarian dietary patterns are associated with a lower risk of metabolic syndrome: the Adventist Health Study 2. Diabetes Care 34, $1225-1227$.

3. Orlich MJ, Jaceldo-Siegl K, Sabate J, et al. (2014) Patterns of food consumption among vegetarians and non-vegetarians. Br J Nutr 112, 1644-1653.

4. Chiu TH, Huang HY, Chiu YF, et al. (2014) Taiwanese vegetarians and omnivores: dietary composition, prevalence of diabetes and IFG. PLOS ONE $\mathbf{9}$, e88547.

5. Blanch N, Clifton PM \& Keogh JB (2014) A systematic review of vascular and endothelial function: effects of fruit, vegetable and potassium intake. Nutr Metab Cardiovasc Dis 25, 253-266.

6. Appel LJ, Moore TJ, Obarzanek E, et al. (1997) A clinical trial of the effects of dietary patterns on blood pressure. DASH Collaborative Research Group. N Engl J Med 336, 1117-1124.

7. Brathwaite N, Fraser HS, Modeste N, et al. (2003) Obesity, diabetes, hypertension, and vegetarian status among SeventhDay Adventists in Barbados: preliminary results. Ethn Dis 13, 34-39.

8. Hung CJ, Huang PC, Lu SC, et al. (2002) Plasma homocysteine levels in Taiwanese vegetarians are higher than those of omnivores. J Nutr 132, 152-158.

9. Lu SC, Wu WH, Lee CA, et al. (2000) LDL of Taiwanese vegetarians are less oxidizable than those of omnivores. J Nutr 130, 1591-1596.

10. Salvini S, Hunter DJ, Sampson L, et al. (1989) Food-based validation of a dietary questionnaire: the effects of week-to-week variation in food consumption. Int J Epidemiol 18, 858-867.

11. Chou Y-C, Chu C-H, Wu M-H, et al. (2011) Dietary intake of vitamin $\mathrm{B}(6)$ and risk of breast cancer in Taiwanese women. J Epidemiol 21, 329-336.

12. Yang Y (2000) The correlation between dietary factors and blood lipids. Master Thesis, School of Public Health, National Defense Medical Center, Taipei, Taiwan.

13. Lee MS, Lai CJ, Yang FY, et al. (2008) A global overall dietary index: ODI-R revised to emphasize quality over quantity. Asia Pac J Clin Nutr 17, Suppl. 1, 82-86.

14. Lee MS, Huang YC, Su HH, et al. (2011) A simple food quality index predicts mortality in elderly Taiwanese. J Nutr Health Aging 15, 815-821.

15. Shang P, Shu Z, Wang Y, et al. (2011) Veganism does not reduce the risk of the metabolic syndrome in a Taiwanese cohort. Asia Pac J Clin Nutr 20, 404-410.

16. Expert Panel on Detection, Evaluation, and Treatment of High Blood Cholesterol in Adults (2001) Executive summary of the third report of The National Cholesterol Education Program (NCEP) expert panel on detection, evaluation, and treatment of high blood cholesterol in adults (Adult Treatment Panel III). JAMA 285, 2486-2497.

17. Pan WH, Yeh WT \& Weng LC (2008) Epidemiology of metabolic syndrome in Asia. Asia Pac J Clin Nutr 17, Suppl. 1, $37-42$.
18. Diggle P, Liang K-Y \& Zeger SL (1994) Analysis of Longitudinal Data, Oxford Statistical Science Series. Oxford, New York: Clarendon Press, Oxford University Press.

19. Wen CP, Wai JP, Tsai MK, et al. (2011) Minimum amount of physical activity for reduced mortality and extended life expectancy: a prospective cohort study. Lancet 378, 1244-1253.

20. Ainsworth BE, Haskell WL, Herrmann SD, et al. (2011) 2011 Compendium of physical activities: a second update of codes and MET values. Med Sci Sports Exerc 43, 1575-1581.

21. Cleveland (1979) Robust locally weighted regression and smoothing scatterplots. J Am Stat Assoc 74, 829-836.

22. Hung CJ, Huang PC, Li YH, et al. (2006) Taiwanese vegetarians have higher insulin sensitivity than omnivores. Br J Nutr $\mathbf{9 5}$, 129-135.

23. Kuo C-S, Lai N-S, Ho L-T, et al. (2004) Insulin sensitivity in Chinese ovo-lactovegetarians compared with omnivores. EurJ Clin Nutr 58, 312-316.

24. Huang D, Dhawan T, Young S, et al. (2011) Fructose impairs glucose-induced hepatic triglyceride synthesis. Lipids Health Dis 10, article no. 20.

25. Ornish D, Brown SE, Scherwitz LW, et al. (1990) Can lifestyle changes reverse coronary heart disease? The Lifestyle Heart Trial. Lancet 336, 129-133.

26. Ornish D, Scherwitz LW, Billings JH, et al. (1998) Intensive lifestyle changes for reversal of coronary heart disease. JAMA 280, 2001-2007.

27. De Biase SG, Fernandes SF, Gianini RJ, et al. (2007) Vegetarian diet and cholesterol and triglycerides levels. Arq Bras Cardiol 88, 35-39.

28. Teixeira Rde C, Molina Mdel C, Zandonade E, et al. (2007) Cardiovascular risk in vegetarians and omnivores: a comparative study. Arq Bras Cardiol 89, 237-244.

29. Toohey ML, Harris MA, DeWitt W, et al. (1998) Cardiovascular disease risk factors are lower in African-American vegans compared to lacto-ovo-vegetarians. J Am Coll Nutr 17, 425-434.

30. Singh PN, Sabate J \& Fraser GE (2003) Does low meat consumption increase life expectancy in humans? Am J Clin Nutr 78, 526S-532S.

31. Appleby PN, Davey GK \& Key TJ (2002) Hypertension and blood pressure among meat eaters, fish eaters, vegetarians and vegans in EPIC-Oxford. Public Health Nutr 5, 645-654.

32. Orlich MJ, Singh PN, Sabate J, et al. (2013) Vegetarian dietary patterns and mortality in Adventist Health Study 2. JAMA Intern Med 173, 1230-1238.

33. Yang FY, Wahlqvist ML \& Lee MS (2008) Body mass index (BMI) as a major factor in the incidence of the metabolic syndrome and its constituents in unaffected Taiwanese from 1998 to 2002. Asia Pac J Clin Nutr 17, 339-351.

34. Mishra S, Xu J, Agarwal U, et al. (2013) A multicenter randomized controlled trial of a plant-based nutrition program to reduce body weight and cardiovascular risk in the corporate setting: the GEICO study. Eur J Clin Nutr 67, 718-724. 